\title{
Human Leukocyte Antigen Class I and Programmed Death-Ligand 1 Coexpression Is an Independent Poor Prognostic Factor in Adenocarcinoma of the Lung
}

Yeon Bi Han ${ }^{1}$. Hyun Jung Kwon ${ }^{1}$ Soo Young Park ${ }^{1}$. Eun-Sun Kim ${ }^{1}$ Hyojin $\mathrm{Kim}^{1} \cdot$ Jin-Haeng Chung ${ }^{1,2}$

'Department of Pathology, Seoul National University Bundang Hospital, Seongnam; 2Department of Pathology, Seoul National University College of Medicine, Seoul, Korea

Received: July 20, 2018

Revised: December 1, 2018

Accepted: December 26, 2018

\section{Corresponding Author}

Jin-Haeng Chung, MD, PhD

Department of Pathology, Seoul National University

Bundang Hospital, 82 Gumi-ro 173beon-gil,

Bundang-gu, Seongnam 13620, Korea

Tel: +82-31-787-7713

Fax: $+82-31-787-4012$

E-mail: chungjh@snu.ac.kr

\begin{abstract}
Background: Both human leukocyte antigen (HLA) class I and programmed death-ligand 1 (PDL1) molecules are known to play important roles in cancer immunity. In this study, we evaluated HLA class I expression in resected adenocarcinoma of the lung, and investigated its prognostic impact in correlation with PD-L1 expression. Methods: HLA class I and PD-L1 expression was evaluated by immunohistochemistry in a total of 403 resected lung adenocarcinomas using tissue microarray. Correlations between the expression of HLA class I/PD-L1 and clinicopathologic features and prognostic significance were analyzed. Results: HLA class I expression was reduced in $91.6 \%$ of adenocarcinoma, and more frequently reduced in patients with younger age, absence of vascular invasion, and low pathologic stage $(p=.033, p=.007$, and $p=.012$, respectively). Positive PD-L1 expression in tumor cells was $16.1 \%$ ( $1 \%$ cut-off), and associated with poor differentiation, presence of vascular invasion and nodal metastasis $(p<.001, p=.002$, and $p=.032$, respectively). On survival analysis, HLA class I or PD-L1 expression alone did not show any statistical significance. On the integrated analysis, HLA class I (+)/PD-L1 (+) subgroup showed a significantly shorter overall survival than other groups $(p=.001)$. Multivariate analysis revealed that coexpression of HLA class I and PD-L1 was an independent poor prognostic factor of lung adenocarcinoma. ( $p<.001$; hazard ratio, 6.106; $95 \%$ confidence interval, 2.260 to 16.501). Conclusions: Lung adenocarcinoma with coexpression of HLA class I and PD-L1 was associated with poor prognosis. This subgroup may evade immune attack by expressing PD-L1 protein despite HLA expression.
\end{abstract}

Key Words: Human leukocyte antigen class I; Programmed cell death-ligand 1; Carcinoma, non-small cell lung; Immunohistochemistry
Lung cancer is a leading cause of cancer-related deaths worldwide. Despite many advances in targeted therapy based on molecular testing, ${ }^{1}$ the 5 -year overall survival (OS) for advanced lung cancer still remains dismal. ${ }^{2}$ Recently, there has been a remarkable progress in immunotherapies for the treatment of non-small cell lung cancer (NSCLC). Immunotherapy with antibodies blocking immune checkpoints, including anti-programmed death ligand-1 (PD-L1), showed durable tumor regression in advanced NSCLCs. ${ }^{3}$ Although PD-L1 expression has emerged as a useful biomarker capable of predicting which patients are more likely to respond to anti-programmed death-1 (PD1)/PD-L1 therapy, PD-L1 expression is not an absolute predictive marker of clinical response. These findings have intrigued researchers to characterize the host's immune response against tumor, as well as tumor's ability to escape the immune attack.

In lung cancer, cytotoxic lymphocytes (CTLs) that play a main role in the anticancer response are actively suppressed in tumor environment. ${ }^{4}$ Two major mechanisms, which affect the target cells and the effector phase of the immune response, play a crucial role in the immune editing process. One is represented by the down-regulation of tumor antigen processing and presentation caused by abnormalities in the human leukocyte antigen (HLA) class I antigen processing machinery (APM). ${ }^{5}$ The other is represented by the anergy of effecter immune cell infiltrates in the tumor microenvironment caused by aberrant inhibitory signals such as immune checkpoint receptor ligands, PD-L1. ${ }^{5}$ These two mechanisms, the expression of PD-L1 and the down-regulation of HLA class I by tumor cells, are also crucial factors for the tumor development process.

Although defects in HLA class I APM components are known to be associated with a reduced OS in various types of cancer, ${ }^{6}$ the prognostic value of HLA class I expression in NSCLC is still controversial. ${ }^{7-9}$ Only limited data are available on the correlation between the expression of PD-L1 and HLA-I in NSCLC. 
To better understand the importance of the PD-L1 and HLA class I expression in lung adenocarcinoma (ADC), we investigated their expression in lung ADC by immunohistochemistry (IHC) and correlated with clinicopathologic features including progression-free survival (PFS) and OS.

\section{MATERIALS AND METHODS}

\section{Patients and samples}

Our cohort consisted of 403 patients with lung ADC who underwent curatively intended surgical resection between May 2003 and December 2012 at Seoul National University Bundang Hospital. None received preoperative chemotherapy or radiation therapy. Clinico-pathological information was obtained from electronic medical records and pathology reports. The pathologic staging was based on the seventh edition of the American Joint Committee on Cancer staging manual. ${ }^{10}$ The study protocol was approved by the Institutional Review Board of Seoul National University Bundang Hospital (B-1704/393-303). Informed consent was waived.

\section{Construction of tissue microarray}

Hematoxylin and eosin $(\mathrm{H} \& \mathrm{E})$-stained slides were reviewed in each case to confirm the original diagnosis and classify the histologic subtype according to 2015 World Health Organization criteria. ${ }^{11}$ The slides were independently reviewed by two pathologists (H. Kim and J.H. Chung) to select the most representative sections. The most representative tumor area was carefully marked on the H\&E-stained slide of each sample tissue. A tissue microarray (TMA) was constructed using 2-mm diameter cores derived from the representative tumor areas selected at random of the formalin-fixed paraffin-embedded tissue blocks from each case by SuperBioChips Laboratories (Seoul, Korea).

\section{Immunohistochemical analysis}

TMAs were sectioned at a thickness of $4-\mu \mathrm{m}$ and stained using an anti-HLA class I ABC antibody (EMR8-5, ab70328, Abcam, Cambridge, MA, USA). Briefly, the slides were stained with an anti-HLA class I ABC (EMR8-5) mouse monoclonal antibody with Ventana BenchMark XT Staining systems (Tucson, AZ, USA). Expressions of HLA class I was assessed by two pathologists (Y.B. Han and J.H. Chung). The expression level of HLA class I on tumor cells was defined as strongly positive ("retained" expression) when tumor cells for which the membrane was strongly and homogenously stained at the same level as stromal lymphocytes or endothelial cells comprised $\geq 75 \%$ of tumor cells (Fig. $1 \mathrm{~A}$ ), weakly positive for $25 \%-74 \%$, or tumor cells with the membrane stained more weakly than stromal lymphocytes or endothelial cells comprising $\geq 25 \%$ (Fig. 1B), and negative when stained cells comprised $<25 \%$ (Fig. 1C). Cases with negative or
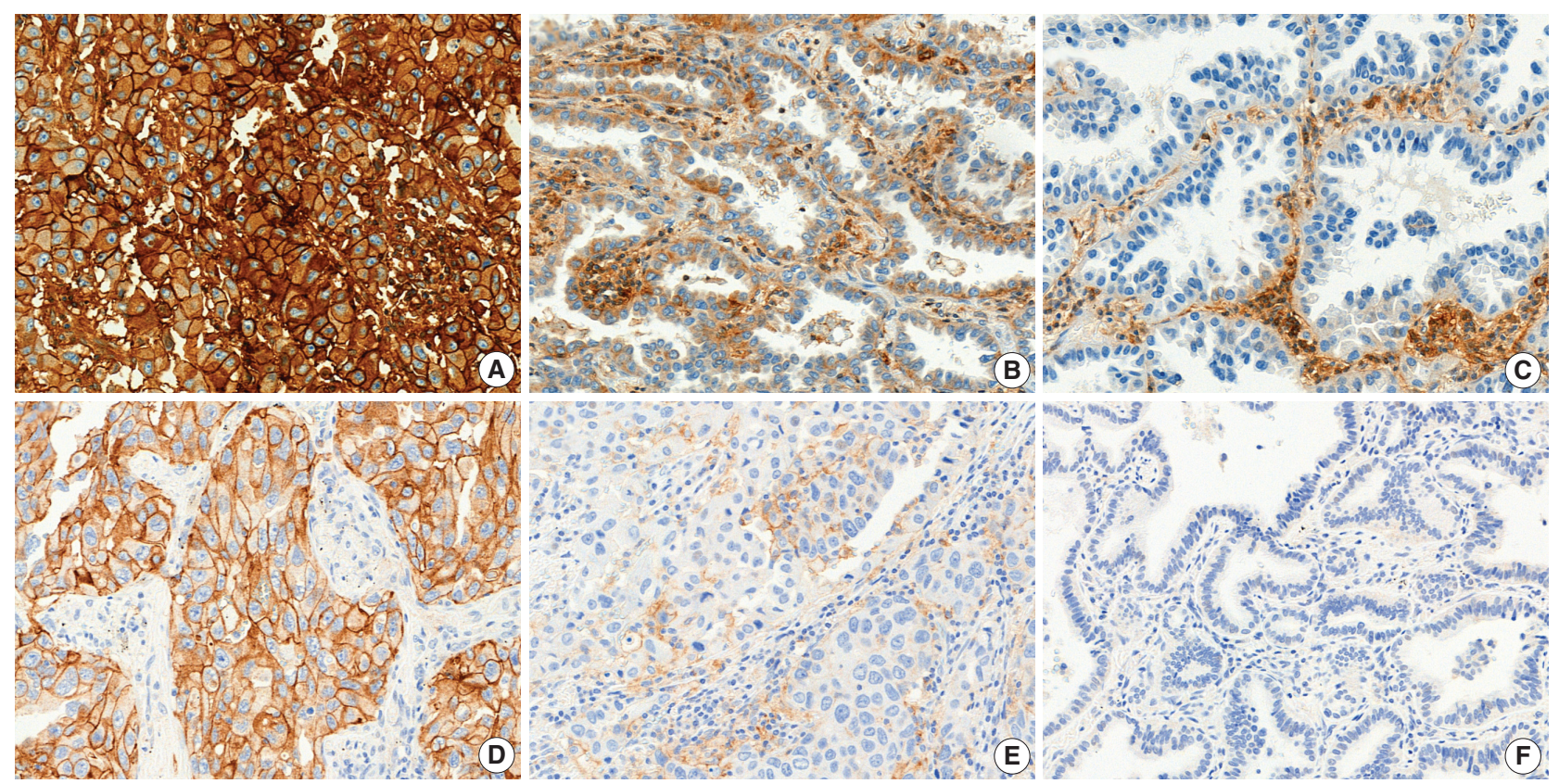

Fig. 1. Human leukocyte antigen (HLA) class I (A-C) and programmed death-ligand 1 (PD-L1) (D-E) expression in lung adenocarcinoma. (A) Strongly positive ("retained") staining of HLA class I in tumor cells. (B) Weakly positive staining of HLA class I. (C) Negative staining of HLA class I. (D) $\geq 50 \%$ positive staining of PD-L1 in tumor cells. (E) 1\%-49\% positive staining of PD-L1. (F) <1\% staining of PD-L1. 
weakly positive staining were judged as representing "reduced" expression.

PD-L1 expression had been evaluated in a previous study with IHC using anti-PD-L1 22C3 mouse monoclonal primary antibody with the EnVision FLEX visualization system on a Dako Autostainer Link 48 system (Carpinteria, CA, USA). ${ }^{12}$ Each tumor cell was judged as positive staining for PD-L1 when the membrane was stained at any intensity. Each patient was classified into "negative" or "positive" according to the percentage of PD-L1 positive tumor cells; two different cut-off values (1\% and 50\%) used on the basis of the published association of this cutoff with anti-PD-1 therapeutic response (Fig. 1D-F). ${ }^{13}$

\section{Statistical analysis}

The chi-square test (or Fisher exact test when appropriate) was used to assess the significance of the association of HLA class I and PD-L1 expression with clinicopathological parameters. A Kaplan-Meier analysis was performed to construct survival curves and statistical significance was assessed using the log-rank test. A multivariate analysis was performed by Cox proportional hazards regression modeling. All statistical tests were two sided and statistical significance was accepted for $\mathrm{p}$-values of $<.05$. All statistical analysis was carried out using Statistical Package for the Social Sciences ver. 21 (IBM Corp., Armonk, NY, USA).

\section{RESULTS}

\section{Clinicopathologic characteristics}

The clinicopathological features and IHC results of the patients are summarized in Table 1 . There was a female predominance in this cohort $(52.6 \%)$. The mean age of patients was 65 years (range, 23 to 82 years) with 242 never smokers (60\%), 89 exsmokers (22.1\%), and 72 current smokers (17.9\%). Regarding pathologic stage, 234 patients were stage I (58.1\%), 83 were stage II (20.6\%), and 86 were (21.3\%) stage III.

\section{HLA class I and PD-L1 expression}

HLA class I expression was observed in the normal bronchial epithelium, type II pneumocytes and other non-neoplastic cells with membranous staining. In the tumor cells, 34 specimens (8.4\%) showed strongly positive membranous expression of HLA class I antigen, 120 specimens (29.8\%) with weakly positive expression, and 249 specimens (61.8\%) with negative expression. Therefore, HLA class I expression was reduced in 369 patients (91.6\%) and retained in 34 patients (8.4\%). Reduced HLA-class I expression was more frequently seen in younger age, absence of
Table 1. Clinicopathologic characteristics

\begin{tabular}{|c|c|}
\hline Characteristic & No. (\%) \\
\hline \multicolumn{2}{|l|}{ Age (yr) } \\
\hline Median (range) & 65 (23-82) \\
\hline \multicolumn{2}{|l|}{ Sex } \\
\hline Male & $191(47.4)$ \\
\hline Female & $212(52.6)$ \\
\hline \multicolumn{2}{|l|}{ Smoking status ${ }^{a}$} \\
\hline Never & $242(60.0)$ \\
\hline Ex-smoker & $89(22.1)$ \\
\hline Current & $72(17.9)$ \\
\hline \multicolumn{2}{|l|}{ Differentiation ${ }^{b}$} \\
\hline Well differentiated & $34(8.4)$ \\
\hline Moderately differentiated & $315(78.2)$ \\
\hline Poorly differentiated & $54(13.4)$ \\
\hline \multicolumn{2}{|l|}{ Genetic status $^{c}$} \\
\hline EGFR mutant & $192(69.1)$ \\
\hline$A L K$ mutant & $20(7.2)$ \\
\hline KRAS mutant & $16(5.8)$ \\
\hline EGFR(-)/ALK(-)/KRAS(-) & $50(18.0)$ \\
\hline \multicolumn{2}{|l|}{ Tumor size $(\mathrm{cm})$} \\
\hline Mean (range) & $31.7(0.8-16.0)$ \\
\hline \multicolumn{2}{|l|}{ Pleural invasion } \\
\hline Absent & $235(58.3)$ \\
\hline Present & $168(41.7)$ \\
\hline \multicolumn{2}{|l|}{ Venous invasion } \\
\hline Absent & $302(74.9)$ \\
\hline Present & $101(25.1)$ \\
\hline \multicolumn{2}{|l|}{ Lymphatic invasion } \\
\hline Absent & $213(52.9)$ \\
\hline Present & $190(47.1)$ \\
\hline \multicolumn{2}{|l|}{ Perineural invasion } \\
\hline Absent & $380(94.3)$ \\
\hline Present & $23(5.7)$ \\
\hline \multicolumn{2}{|l|}{ Pathologic stage } \\
\hline IA & $127(31.5)$ \\
\hline IB & $107(26.6)$ \\
\hline$\| \mathrm{A}$ & $64(15.9)$ \\
\hline$\| \mathrm{B}$ & $19(4.7)$ \\
\hline$\| \mathrm{A}$ & $77(19.1)$ \\
\hline IIIB & $9(2.2)$ \\
\hline \multicolumn{2}{|l|}{ HLA class I IHC (\%) } \\
\hline$<25$ & $249(61.8)$ \\
\hline $25-74$ & $120(29.8)$ \\
\hline$\geq 75$ & $34(8.4)$ \\
\hline \multicolumn{2}{|l|}{ PD-L1 IHC (\%) } \\
\hline$<1$ & 338 (83.9) \\
\hline $1-49$ & $42(10.4)$ \\
\hline$\geq 50$ & $23(5.7)$ \\
\hline Total & $403(100)$ \\
\hline
\end{tabular}

EGFR, epidermal growth factor receptor; ALK, anaplastic lymphoma kinase; HLA, human leukocyte antigen; IHC, immunohistochemistry; PD-L1, programmed death-ligand 1.

aSmoking status was defined as follows: never smoker $(<100$ cigarettes per lifetime); ex-smoker ( $\geq 100$ cigarettes per lifetime and quit $>1$ year prior to the diagnosis); current smoker ( 2100 cigarettes per lifetime and smoked at the time of lung cancer diagnosis or quit $\leq 1$ year prior to the diagnosis); ${ }^{b}$ Differentiation was defined as follows: well differentiated (lepidic predominant); moderately differentiated (acinar or papillary predominant); poorly differentiated (micropapillary or solid predominant); ' Genetic status was evaluable for 278 patients. 
vascular invasion, and low pathologic stage $(\mathrm{p}=.033, \mathrm{p}=.007$, and $\mathrm{p}=.012$, respectively). PD-L1 expression was normally expressed in membranes of the alveolar macrophages and some lympho- cytes. In the tumor cells, 65 patients $(16.1 \%)$ with cutoff value of $1 \%$ and 23 patients $(5.7 \%)$ with cutoff value of $50 \%$ showed positive PD-L1 expression. Positive PD-L1 expression (1\% cutoff)

Table 2. HLA class I, PD-L1 expression and clinicopathologic characteristics

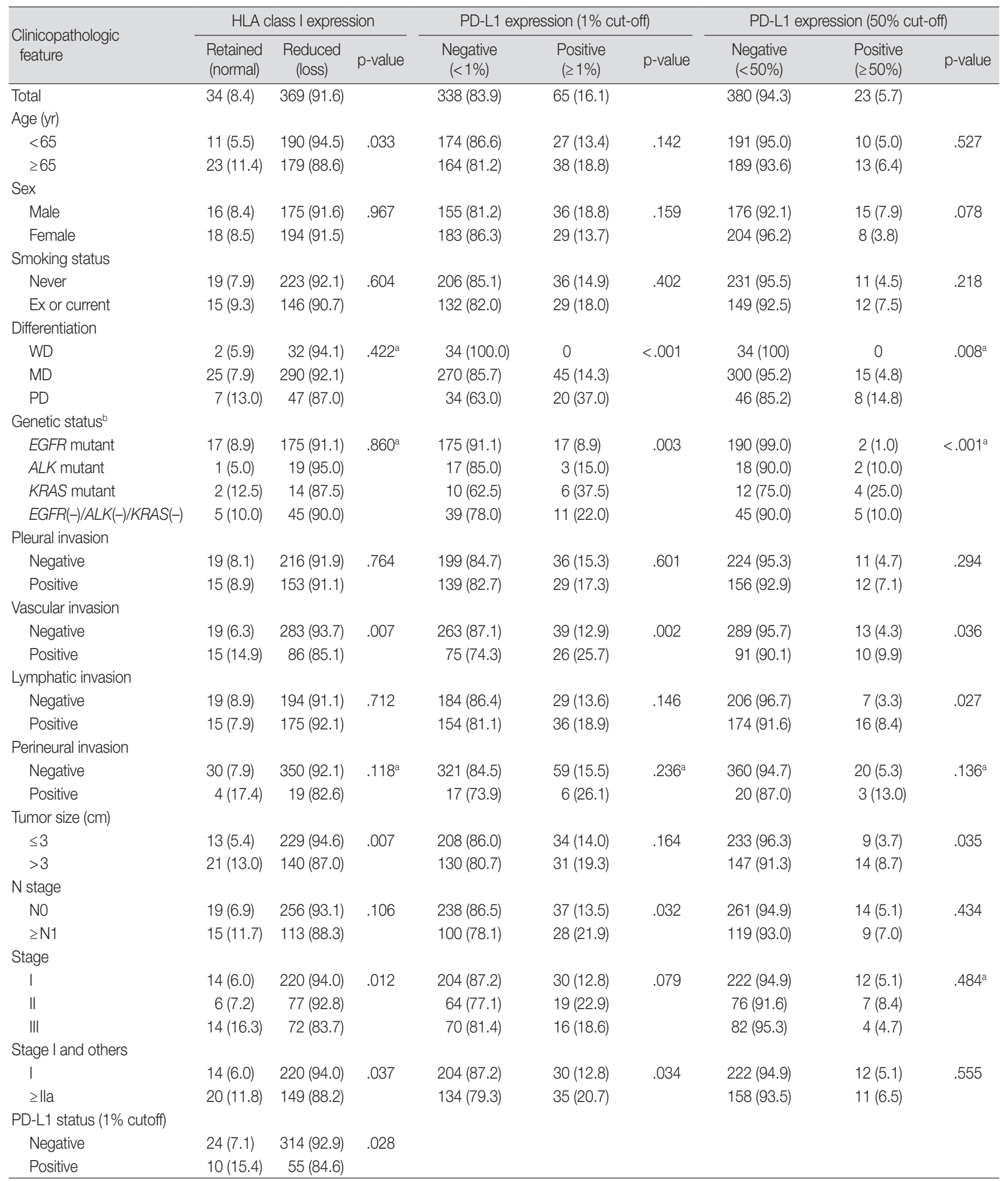

${ }^{a} p$-value was obtained by Fisher exact test; ${ }^{b}$ Genetic status was evaluable for 278 patients. 
was associated with poor differentiation, presence of vascular invasion and nodal metastasis $(\mathrm{p}<.001, \mathrm{p}=.002$, and $\mathrm{p}=.032$, respectively). In addition, positive PD-L1 expression (1\% cutoff) was correlated with epidermal growth factor receptor (EGFR) wild type tumors $(\mathrm{p}=.003)$ (Table 2$)$.

\section{Survival analysis}

At the time of analysis, the median PFS was 36.0 months and the median OS was 65.0 months. During this time, 164 patients (40.7\%) suffered tumor recurrence and 90 patients $(22.3 \%)$ died due to cancer. Survival analysis using Kaplan-Meier and Cox proportional hazards analyses were performed to evaluate the prognostic impact of HLA class I and PD-L1 protein expression. There was no significant difference in OS according to HLA class I status (5-year survival, $81.4 \%$ in "reduced" patients vs. $69.3 \%$ in "retained" patients; $\mathrm{p}=.115$ ) (Fig. 2A).

There was no significant difference in OS according to PD-L1 expression status, either. (Fig. 2B). The 5-year OS of PD-L1positive and PD-L1-negative patients using each cutoff value was $75.4 \%$ and $81.3 \%$ (cutoff, $1 \% ; \mathrm{p}=.423$ ), $78.7 \%$ and $80.5 \%$ (cutoff, $50 \% ; \mathrm{p}=.534$ ), respectively. However, when both markers were analyzed simultaneously, coexpression of HLA class I and PD-L1 (1\% cutoff) group $(\mathrm{n}=10)$ had a significantly shorter OS than other groups (5-year survival, 42.0\%; $\mathrm{p}=.009)($ Fig. 2C).
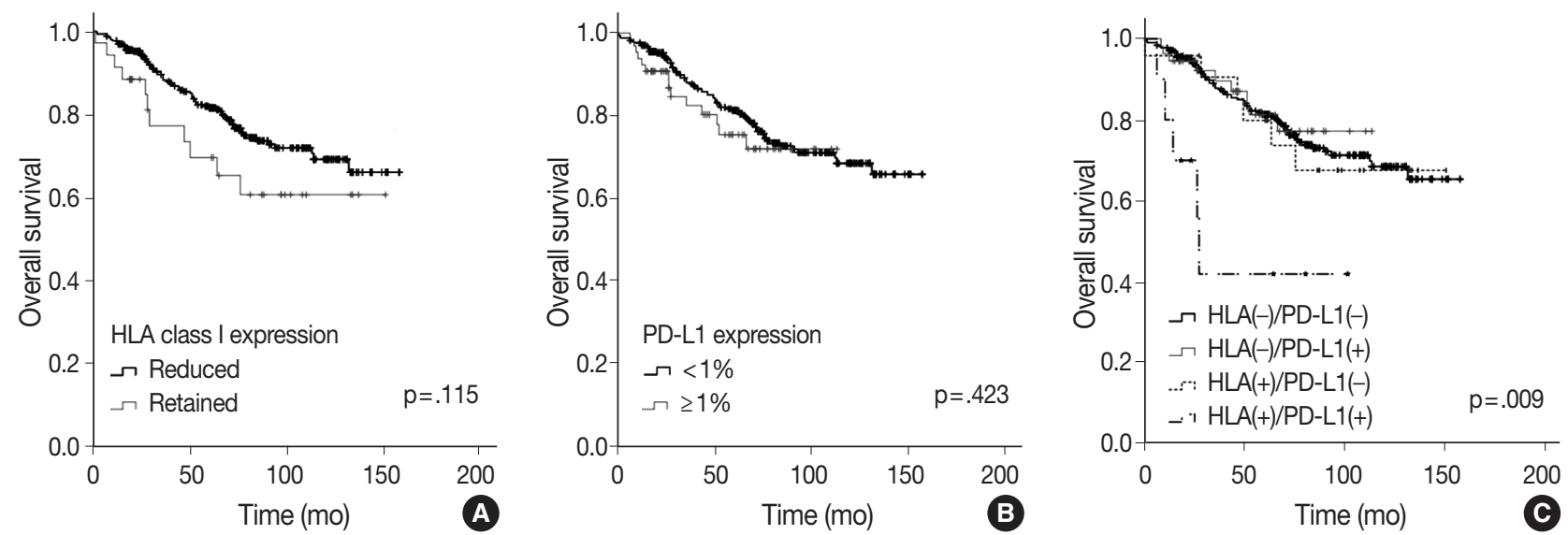

Fig. 2. Association of human leukocyte antigen $(\mathrm{HLA})$ class I antigen and programmed death-ligand 1 (PD-L1) expression in lung adenocarcinoma (ADC) lesions with overall survival in patients. (A) Overall survival curves of patients with lung ADC according to their HLA class I expression. (B) Overall survival curves according to PD-L1 expression status classified by the percentage of tumor cells expressing PD-L1 at the cutoff value of $1 \%$. (C) Overall survival in patients classified by expression of HLA class I and PD-L1. Patients were divided into four groups: HLA class I (-)/PD-L1 (-), HLA class I (-)/PD-L1 (+), HLA class I (+)/PD-L1 (-), HLA class I (+)/PD-L1 (+).
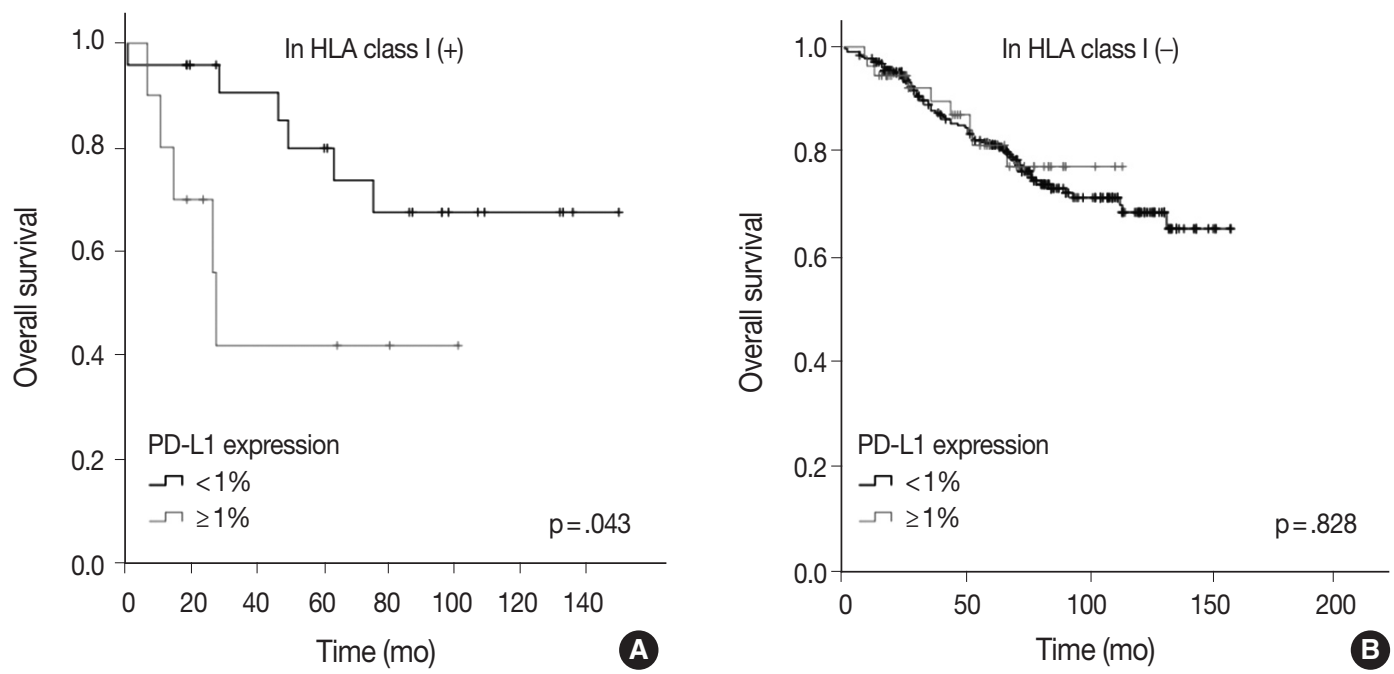

Fig. 3. Overall survival according to programmed death-ligand 1 (PD-L1) expression after stratification by human leukocyte antigen (HLA) class I expression status. (A) Overall survival curves according to PD-L1 expression status (1\% cutoff) among "retained" HLA class I cases. (B) Overall survival curves according to PD-L1 expression status (1\% cutoff) among "reduced" HLA class I cases. 
When analyzed by PD-L1 expression with stratification by HLA class I status, a significantly poor prognosis in PD-L1positive patients (1\% cutoff) was documented only when HLA class I expression was "retained" ( $\mathrm{n}=34, \mathrm{p}=.043$ ) (Fig. 3A). Among patients with "reduced" HLA class I expression, PD-L1 expression status provided no prognostic impact ( $\mathrm{p}=.828$ ) (Fig. 3B).

In addition, there was no significant difference in PFS according to HLA class I status and PD-L1 status. Multivariate analysis using Cox's proportional hazards model revealed coexpression of HLA class I and PD-L1 as an independent factor associated with poor prognosis $(\mathrm{p}<.001$; hazard ratio, $6.106 ; 95 \%$ confidence

Table 3. Univariate and multivariate analyses of clinicopathologic factors and overall survival in lung ADC patients

\begin{tabular}{|c|c|c|c|}
\hline \multirow{2}{*}{ Clinicopathologic variable } & \multirow{2}{*}{$\frac{\text { Univariate }}{p \text {-value }}$} & \multicolumn{2}{|r|}{ Multivariate } \\
\hline & & $p$-value & $\mathrm{HR}(95 \% \mathrm{Cl})$ \\
\hline \multicolumn{4}{|l|}{ Age } \\
\hline$<65$ yr vs $\geq 65 \mathrm{yr}$ & $<.001$ & $<.001$ & $2.871(1.715-4.804)$ \\
\hline \multicolumn{4}{|l|}{ Sex } \\
\hline Male vs female & $<.001$ & & \\
\hline \multicolumn{4}{|l|}{ Smoking $\mathrm{Hx}$} \\
\hline Never vs ever or current & .025 & .026 & $1.774(1.073-2.935)$ \\
\hline \multicolumn{4}{|l|}{ Differentiation } \\
\hline WD vs MD vs PD & .077 & - & - \\
\hline \multicolumn{4}{|l|}{ Genetic status } \\
\hline $\begin{array}{l}\text { EGFR mutant vs ALK mutant } \\
\text { vs KRAS mutant vs wild type }\end{array}$ & .179 & - & - \\
\hline \multicolumn{4}{|l|}{ Pleural invasion } \\
\hline Negative vs positive & $<.001$ & - & - \\
\hline \multicolumn{4}{|l|}{ Vascular invasion } \\
\hline Negative vs positive & .002 & - & - \\
\hline \multicolumn{4}{|l|}{ Lymphatic invasion } \\
\hline Negative vs positive & $<.001$ & - & - \\
\hline \multicolumn{4}{|l|}{ Perineural invasion } \\
\hline Negative vs positive & $<.001$ & - & - \\
\hline \multicolumn{4}{|l|}{ Tumor size } \\
\hline$\leq 3 \mathrm{~cm}$ vs $>3 \mathrm{~cm}$ & $<.001$ & - & - \\
\hline \multicolumn{4}{|l|}{$\mathrm{N}$ category } \\
\hline No vs $\geq N 1$ & $<.001$ & .122 & $2.143(0.815-5.632)$ \\
\hline \multicolumn{4}{|l|}{ Stage } \\
\hline | vs || vs ||| & $<.001$ & .039 & \\
\hline | vs || & & .294 & $1.732(0.621-4.827)$ \\
\hline | vs ||| & & .027 & 3.295 (1.142-9.508) \\
\hline \multicolumn{4}{|l|}{ HLA expression } \\
\hline$<75 \%$ vs $\geq 75 \%$ & .115 & - & - \\
\hline \multicolumn{4}{|l|}{ PD-L1 expression } \\
\hline$<1 \%$ vs $\geq 1 \%$ & .423 & - & - \\
\hline \multicolumn{4}{|l|}{ HLA(+)/PD-L1(+) } \\
\hline Others vs double positive & .001 & $<.001$ & $6.106(2.260-16.501)$ \\
\hline
\end{tabular}

$\mathrm{ADC}$, adenocarcinoma; $\mathrm{HR}$, hazard ratio; $\mathrm{Cl}$, confidence interval; $\mathrm{Hx}$, history; $W D$, well differentiated; $M D$, moderately differentiated; $P D$, poor differentiated; EGFR, epidermal growth factor receptor; ALK, anaplastic lymphoma kinase; HLA, human leukocyte antigen; PD-L1, programmed death-ligand 1. aWild type defined as EGFR(-)/ALK(-)/KRAS(-). interval, 2.260 to 16.501) (Table 3).

\section{DISCUSSION}

In this study, we evaluated the expression of HLA class 1 and PD-L1 protein in 403 lung ADCs and investigated the relationship between HLA class 1/PD-L1 protein expression and various clinicopathological factors and molecular characteristics. Our study showed that HLA class I expression was reduced in $91.6 \%$, and positive PD-L1 expression was $16.1 \%$ (1\% cutoff) in surgically resected lung ADC. On survival analysis, HLA class I or PD-L1 expression alone did not showed any statistical significance. On the integrated analysis, HLA class I (+)/PD-L1 (+) subgroup showed a significant shorter OS than other groups $(\mathrm{p}=.009)$. Multivariate analysis revealed that coexpression of HLA class I and PD-L1 was an independent poor prognostic factor of lung ADC ( $<$ <.001; hazard ratio, 6.106; 95\% confidence interval, 2.260 to 16.501$)$.

The frequency of HLA class I antigen reduction in this cohort seemed to be high compared to other studies for NSCLC ( $43 \%$ to $93.6 \%){ }^{7-9,14}$ Hirai et al. ${ }^{7}$ reported that reduced HLA class I expression was observed in $43 \%$ of the stage I lung ADC, and they used $20 \%$ and $80 \%$ cutoff. Ramnath et al. ${ }^{9}$ reported that reduced HLA class I expression was observed in $93.6 \%$ of the NSCLC patients, and they used $25 \%$ and $75 \%$ cutoff. CTLs can recognize tumor-specific antigens presented on various types of HLA class I molecules on the tumor. ${ }^{15,16}$ As the prerequisite of anti-tumor activity of CTLs is the recognition of immunogenic epitopes presented on HLA class I molecules on the tumor cells, reduced expression of HLA class I molecule in lung ADC is not surprising. However, the prognostic impact of HLA class I molecule expression is highly controversial.

High HLA class I expression had been reported to be associated with a better survival in esophageal cancer, hepatocellular carcinoma and pancreatic cancer, ${ }^{17-19}$ or poor prognosis in gastric cancer and colorectal cancer, ${ }^{16,20,21}$ These contradictory results might be explained by multilateral function of HLA class I molecules. As HLA-B/C molecule has been known to act as an inhibitory receptor against natural killer (NK) cells, cancer cells with loss of HLA class I molecule could be attacked by NK cells. $^{15,16}$ The prognostic impact of HLA class I molecule expression could be variable depending on the mechanism of activation of the immune system in each organ. In the present study, there was no significant correlation between HLA class I expression status and survival, which may reflect HLA class I antigen's diverse roles in immune system. 
In this cohort, positive PD-L1 expression was associated with poor differentiation, wild type EGFR, presence of vascular invasion and nodal metastasis, which is consistent with previous reports. ${ }^{7,12,22}$ However, there was no significant difference between PD-L1 expressions and survival. Even though neither HLA class I nor PD-L1 alone was associated with the clinical outcome of the patients, integrated analysis of revealed that coexpression of HLA class I and PD-L1 was significantly associated with poor OS. While the patients with PD-L1 (+)/HLA class $1(+)$ showed worse prognosis, PD-L1 (+)/HLA class 1 (-) group had no significant prognostic impact.

These results might be explained as below.

(1) The tumor cells with retained HLA class I expression can inhibit NK cells and evade immune system by expressing PD-L1. The immunosurveillance of CD8 T cells may be more critical for patients with high HLA class I expression than for patients who express it at a low level. (2) The tumor cells with reduced HLA class I expression may evade immune attack regardless of PD-L1 expression on tumor cells, which may account for the lack of prognostic impact of PD-L1 status. Nevertheless, tumor cells with HLA class I expression can be recognized by CTLs and may evade immune attack by expressing PD-L1, which also may account for the result that PD-L1 positivity was associated with a poor prognosis in tumors with normal HLA class I expression. The exact role of immune mechanism of the HLA class 1 molecule on the pulmonary ADC needs further functional studies. However, we assessed HLA class I status by only antiHLA class I ABC antibody and the lack of available data concerning clinical response to anti-PD-1/PD-L1 therapy represents a major limitation of our study.

In conclusion, coexpression of HLA class I and PD-L1 was associated with poor prognosis in lung ADC. Because coexpression of HLA class I and PD-L1 was an independent prognostic factor for an extremely poor outcome, postoperative patients with HLA class $1(+) /$ PD-L1 (+) should be recommended for short interval follow-up and observation.

\section{ORCID}

Yeon Bi Han: https://orcid.org/0000-0002-3657-5012

Hyun Jung Kwon: https://orcid.org/0000-0001-8822-7899

Soo Young Park: https://orcid.org/0000-0002-1469-2787

Eun-Sun Kim: https://orcid.org/0000-0003-3394-2646

Hyojin Kim: https://orcid.org/0000-0001-9201-8328

Jin-Haeng Chung: https://orcid.org/0000-0002-6527-3814

\section{Author Contributions}

Conceptualization: JHC.

Data curation: $\mathrm{YBH}$.

Formal analysis: HK, JHC.

Funding acquisition: JHC.

Investigation: $\mathrm{YBH}$.

Methodology: YBH, SYP, ESK, HJK, HK, JHC.

Project administration: JHC.

Resources: JHC.

Supervision: JHC.

Validation: YBH, JHC.

Visualization: YBH, JHC.

Writing—original draft: YBH, JHC.

Writing_review \& editing: YBH, SYP, HJK, HK, JHC.

\section{Conflicts of Interest}

The authors declare that they have no potential conflicts of interest.

\section{Acknowledgments}

This study was supported by Research Grants from Seoul National University Bundang Hospital (SNUBH 14-2018018), NRF Grant funded by the Korean Government (MSIT) (No.2017R1A5A1015626) and the Korea Healthcare Technology R\&D Project, Ministry of Health \& Welfare, Republic of Korea (HI17C1290).

\section{REFERENCES}

1. Shim HS, Choi YL, Kim L, et al. Molecular testing of lung cancers. J Pathol Transl Med 2017; 51: 242-54.

2. Torre LA, Sauer AM, Chen MS Jr, Kagawa-Singer M, Jemal A, Siegel RL. Cancer statistics for Asian Americans, Native Hawaiians, and Pacific Islanders, 2016: converging incidence in males and females. CA Cancer J Clin 2016; 66: 182-202.

3. Somasundaram A, Burns TF. The next generation of immunotherapy: keeping lung cancer in check. J Hematol Oncol 2017; 10: 87.

4. Domagala-Kulawik J. The role of the immune system in non-small cell lung carcinoma and potential for therapeutic intervention. Transl Lung Cancer Res 2015; 4: 177-90.

5. Concha-Benavente F, Srivastava R, Ferrone S, Ferris RL. Immunological and clinical significance of HLA class I antigen processing machinery component defects in malignant cells. Oral Oncol 2016; 58: 52-8.

6. Campoli M, Ferrone S. HLA antigen changes in malignant cells: epigenetic mechanisms and biologic significance. Oncogene 2008; 


\section{7: 5869-85}

7. Hirai A, Yoneda K, Shimajiri S, et al. Prognostic impact of programmed death-ligand 1 expression in correlation with human leukocyte antigen class I expression status in stage I adenocarcinoma of the lung. J Thorac Cardiovasc Surg 2018; 155: 382-92.e1.

8. Kikuchi E, Yamazaki K, Torigoe T, et al. HLA class I antigen expression is associated with a favorable prognosis in early stage nonsmall cell lung cancer. Cancer Sci 2007; 98: 1424-30.

9. Ramnath N, Tan D, Li Q, et al. Is downregulation of MHC class I antigen expression in human non-small cell lung cancer associated with prolonged survival? Cancer Immunol Immunother 2006; 55: 891-9.

10. Edge SB, Byrd DR, Compton CC, Fritz AG, Greene FL, Trotti A. AJCC cancer staging manual. 7th ed. New York: Springer, 2010.

11. Travis WD, Brambilla E, Nicholson AG, et al. The 2015 World Health Organization classification of lung tumors: impact of genetic, clinical and radiologic advances since the 2004 classification. J Thorac Oncol 2015; 10: 1243-60.

12. Kim H, Kwon HJ, Park SY, Park Y, Park E, Chung JH. Clinicopathological analysis and prognostic significance of programmed cell death-ligand 1 protein and mRNA expression in non-small cell lung cancer. PLoS One 2018; 13: e0198634.

13. Herbst RS, Baas P, Kim DW, et al. Pembrolizumab versus docetaxel for previously treated, PD-L1-positive, advanced non-small-cell lung cancer (KEYNOTE-010): a randomised controlled trial. Lancet 2016; 387: 1540-50.

14. Perea F, Sánchez-Palencia A, Gómez-Morales M, et al. HLA class I loss and PD-L1 expression in lung cancer: impact on T-cell infiltration and immune escape. Oncotarget 2018; 9: 4120-33.

15. Campbell KS, Purdy AK. Structure/function of human killer cell immunoglobulin-like receptors: lessons from polymorphisms, evolution, crystal structures and mutations. Immunology 2011; 132: 315-25.

16. Ljunggren HG, Kärre K. In search of the 'missing self': MHC molecules and NK cell recognition. Immunol Today 1990; 11: 237-44.

17. Imai D, Yoshizumi T, Okano S, et al. The prognostic impact of programmed cell death ligand 1 and human leukocyte antigen class I in pancreatic cancer. Cancer Med 2017; 6: 1614-26.

18. Mizukami Y, Kono K, Maruyama T, et al. Downregulation of HLA Class I molecules in the tumour is associated with a poor prognosis in patients with oesophageal squamous cell carcinoma. Br J Cancer 2008; 99: 1462-7.

19. Umemoto Y, Okano S, Matsumoto Y, et al. Prognostic impact of programmed cell death 1 ligand 1 expression in human leukocyte antigen class I-positive hepatocellular carcinoma after curative hepatectomy. J Gastroenterol 2015; 50: 65-75.

20. Menon AG, Morreau H, Tollenaar RA, et al. Down-regulation of HLA-A expression correlates with a better prognosis in colorectal cancer patients. Lab Invest 2002; 82: 1725-33.

21. Ueda Y, Ishikawa K, Shiraishi N, Yokoyama S, Kitano S. Clinical significance of HLA class I heavy chain expression in patients with gastric cancer. J Surg Oncol 2008; 97: 451-5.

22. Zhang M, Li G, Wang Y, et al. PD-L1 expression in lung cancer and its correlation with driver mutations: a meta-analysis. Sci Rep 2017; 7: 10255 